\title{
Socio-demographic and Comorbidity Profiles of Migraine Patients in a Headache Clinic of a Tertiary Care Hospital in Dhaka City
}

\author{
Mohammad Akter Hossain', Maliha Hakim², Mashfiqul Hasan³, Mohammad Atiqur Rahman', \\ Mamnur Rashid ${ }^{5}$, Golam Sagir 6 , Mohammad Enayet Hussain
}

${ }^{1}$ Assistant Professor, Department of Neurology, National Institute of Neurosciences and Hospital, Dhaka, Bangladesh; ${ }^{2}$ Professor,

Department of Neurology, National Institute of Neurosciences and Hospital, Dhaka, Bangladesh; ${ }^{3}$ Assistant Registrar, Department of Neurology, National Institute of Neurosciences and Hospital, Dhaka, Bangladesh; ${ }^{4}$ Medical Officer, Department of Neurology, National Institute of Neurosciences and Hospital, Dhaka, Bangladesh; ${ }^{5}$ Junior Consultant, Department of Neurology, National Institute of Neurosciences and Hospital, Dhaka, Bangladesh; ${ }^{6}$ Junior Consultant (Medicine), National Institute of Neurosciences and Hospital, Dhaka, Bangladesh; ${ }^{7}$ Associate Professor, Department of Neurology, National Institute of Neurosciences \& Hospital, Dhaka, Bangladesh;

[Received: 21 January 2016; Revised: 6 March 2016; Accepted: 11 December 2016; Published: 1 January 2017]

\begin{abstract}
Background: Comorbidities of migraine patients are not uncommon. Objective: This study was done to find out sociodemographic characteristics and the important comorbid psychiatric and physical illness in migraine patients. Methodology: This cross sectional study was carried out in the Neurology Outdoor and Headache Clinic at Dhaka Medical College Hospital, Dhaka from July 2010 to June 2011 for a period of 1 year. Patients with migraine (on the basis of International headache society migraine headache criteria), age $\geq 16$ years with total duration of headache six months or more, having comorbidities like major depressive disorder (MDD), generalized anxiety disorder (GAD), hypertension (HTN), diabetes mellitus (DM) or obesity were included in the study $(\mathrm{n}=33)$. The sociodemographic and relevant data were collected in a preformed data sheet from each patient. Result: The mean age of the participants was 33.8 \pm 8.8 years. Most of them were in 31-40 year age group (39.4\%), followed by 21-30 year age group (36.4\%). Most of the participants were female (72.7\%) and housewives $(57.6 \%)$. The symptoms of migraine was severe according to Migraine Severity Scale (MIGSEV) in $19(57.6 \%)$, moderate in $8(24.2 \%)$ and mild in $6(18.2 \%)$ participants. Among the comorbidities, psychiatric problems like GAD and MDD was present in $8(24.2 \%)$ and $6(18.2 \%)$ participants respectively. Seven $(21.2 \%)$ were obese, $4(12.1 \%)$ hypertensive and $8(24.2 \%)$ diabetic. Frequency of headache/month was observed to be higher in participants with hypertension (5.0 \pm 1.4 episodes/month) and duration of episodic migraine headache was higher in those with MDD (23.4 \pm 10.3 hours). Conclusion: Patients of migraine have comorbidities like GAD, MDD, obesity, hypertension and DM which need to be addressed appropriately for proper migraine headache management. [Journal of National Institute of Neurosciences Bangladesh, 2017;3(1): 48-51]
\end{abstract}

\section{Keywords: Migraine; comorbidities; socio-demography}

Correspondence: Dr. Mohammad Akter Hossain, Assistant Professor, Department of Neurology, National Institute of Neurosciences and Hospital, Sher-E-Bangla Nagar, Agargaon, Dhaka, Bangladesh; Email: dr.akter1972@gmail.com; Cell no.: $+8801720848049$

Conflict of interest: There is no conflict of interest relevant to this paper to disclose.

Funding agency: This research project was not funded by any group or any institution.

Contribution to authors: MAH was involved in protocol preparation, data collection and literature search up to manuscript writing. $\mathrm{MH}, \mathrm{MAH}, \mathrm{MH}, \mathrm{MR} \& \mathrm{MEH}$ were involved in manuscript preparation. GS was involved in statistical analysis and involved in literature search up and manuscript writing.

How to cite this article: Hossain MA, Hakim M, Hasan M, Rahman MA, Rashid M, Sagir G, Hussain ME. Socio-demographic and Comorbidity Profiles of Migraine Patients in a Headache Clinic of a Tertiary Care Hospital in Dhaka City. J Natl Inst Neurosci Bangladesh, 2017;3(1): 48-51

Copyright: (C2017 Hossain et al. Published by Journal of National Institute of Neurosciences Bangladesh. This article is published under the Creative Commons CC BY-NC License (https://creativecommons.org/licenses/by-nc/4.0/). This license permits use, distribution and reproduction in any medium, provided the original work is properly cited, and is not used for commercial purposes.

\section{Introduction}

Migraine is a highly prevalent and largely familial disorder characterized by periodic, commonly unilateral, often pulsatile headaches that begin in childhood, adolescence, or early adult life and recur with diminishing frequency during advancing years ${ }^{1}$. 
Comorbidities of migraine patients are not uncommon. American Migraine Prevalence and Prevention (AMPP) study observed that episodic migraine patients suffered from anxiety $(18.77 \%)$, depression $(25.62 \%)$, high blood pressure $(27.82 \%)$ and obesity $(20.95 \%)^{2}$. Epidemiologic studies have reported associations between migraine and psychiatric disorder, primarily major depression and anxiety disorder. Abnormalities related to the neurotransmitter serotonin have been suggested as a neurochemical basis for migraine as well as for major depression ${ }^{3}$.

World-wide many studies were done regarding common comorbidities of migraine patients. The term 'comorbidity', coined by Feinstein, is now widely used to refer to the greater than coincidental association of two conditions in the same individual ${ }^{4}$. Comorbidity can be important for the diagnosis of headache disorders. For a significant proportion of migraine sufferers who seek help for their headaches, diagnosis can be complicated by other conditions that occur simultaneously with migraine. Depression can cause changes in mood or behavior, in addition to head pain-symptoms which are commonly seen with migraine. These overlaps have important implications for the diagnosis of headache conditions-reinforcing the importance of accurate, detailed discussions of symptoms with a physician. In these circumstances, this study was done to find out sociodemographic characteristics and the important comorbid psychiatric and physical illness in migraine patients which might influence both severity and frequency of migraine thus affecting proper management of migraine headache.

\section{Methodology}

This cross-sectional study was carried out in the Neurology Outdoor and Headache Clinic at Dhaka Medical College Hospital, Dhaka from July 2010 to June 2011 for a period of 1 year. The patients were selected on the basis of International headache society (IHS) migraine headache criteria ${ }^{5}$. Patients with the typical history of migraine, age 16 years and above with total duration of headache six months or more, having comorbidities like major depressive disorder (MDD), generalized anxiety disorder (GAD), hypertension (HTN), diabetes mellitus (DM) or obesity were selected as study population. Headache due to causes other than migraine were excluded from this study. Diagnosis of associated psychiatric and physical comorbidities was done on the basis of history, clinical examination and relevant investigations. The short-form version of the Depression Anxiety Stress
Scales (DASS), which is a valid set of three self-report scales having 21(twenty one) items designed to measure the negative emotional states of depression, anxiety and stress, were used in this study ${ }^{6}$. After assessing, depression and anxiety were categorized to major depressive episode and generalized anxiety disorder on the basis of diagnostic criteria of Diagnostic and Statistical Manual of mental disorders. ${ }^{7}$ Persons were diagnosed as diabetes mellitus (DM) according to the diagnostic criteria of American Diabetes Association. Study subjects were diagnosed as a case of hypertension ${ }^{8}$ and obesity ${ }^{9}$ according to the standard diagnostic criteria. The clinical features, investigation findings and relevant data were collected in a preformed data sheet from each patient. Prior to the commencement of this study, the research protocol was approved by the Research review committee (RRC) of Dhaka medical college hospital (DMCH), Dhaka. Informed written consent was taken from each participant. Analysis of data was done by SPSS version 16.0 software.

\section{Results}

This study included 33 patients of migraine headache with various comorbidities. The mean age of the participants was $33.8 \pm 8.8$ years. Most of them were in

Table 1: Characteristics of the Participants $(n=33)$

\begin{tabular}{lcc}
\hline Parameters & Frequency & Percentage \\
\hline Age Group & & \\
16 to 20 Years & 2 & 6.1 \\
21 to 30 Years & 12 & 36.4 \\
31 to 40 Years & 13 & 39.4 \\
41 to 50 Years & 5 & 15.2 \\
$>50$ Years & 1 & 3.0 \\
Mean \pm SD & $33.8 \pm 8.8$ & $33.8 \pm 8.8$ \\
Range (min-max) & $(16-55)$ & $(16-55)$ \\
Sex & & \\
- Male & 9 & 27.3 \\
- Female & 24 & 72.7 \\
Occupation & & \\
- Housewife & 19 & 57.6 \\
- Service holder & 5 & 15.2 \\
- Student & 4 & 12.1 \\
- Business & 5 & 15.2 \\
Migraine severity* & & \\
- Mild & 6 & 18.2 \\
- Moderate & 8 & 24.2 \\
- Severe & 19 & 57.6 \\
\hline
\end{tabular}

*According to migraine severity (MIGSEV) scale 
31-40 year age group (39.4\%), followed by $21-30$ year age group (36.4\%). Most of the participants were female $(72.7 \%)$ and housewives (57.6\%). The symptoms of migraine was severe according to Migraine Severity Scale (MIGSEV) in 19 (57.6\%), moderate in $8(24.2 \%)$ and mild in $6(18.2 \%)$ participants (Table 1).

Table 2: Distribution of various comorbidities along with frequency of headache (per month) and duration of episodic migraine headache (in hours) in study participants $(\mathrm{n}=33)$

\begin{tabular}{lccc}
\hline Comorbidities & n (\%) & $\begin{array}{c}\text { Frequency of } \\
\text { headache/month }\end{array}$ & $\begin{array}{c}\text { Duration of } \\
\text { episodic migraine } \\
\text { headache in hours }\end{array}$ \\
\hline GAD & $8(24.2)$ & $4.7 \pm 1.8$ & $22.2 \pm 10.9$ \\
MDD & $6(18.2)$ & $4.8 \pm 2.0$ & $23.4 \pm 10.3$ \\
Obesity & $7(21.2)$ & $4.8 \pm 1.7$ & $21.4 \pm 10.5$ \\
HTN & $4(12.1)$ & $5.0 \pm 1.4$ & $20.3 \pm 8.9$ \\
DM & $8(24.2)$ & $3.3 \pm 2.2$ & $13.5 \pm 9.3$ \\
\hline
\end{tabular}

GAD: Generalized anxiety disorder; MDD: Major depressive disorder; HTN: Hypertension; DM: Diabetes mellitus

\section{Discussion}

This study aimed to observe frequency of different common psychiatric and physical comorbidities in migraine patient and also noted the sociodemographic profile of the participants. It was observed that GAD and MDD were the frequent psychiatric comorbidity, while diabetes, hypertension and obesity were the common physical comorbidities in patients with migraine.

Majority of the participants of this study were in their 4th decade of life. But, Habib et allo observed that, $40.27 \%$ of migraine patients were within the age range of 18 to 29 years. It might be important to address the comorbidities in older patients to improve treatment outcome. Migraine is more common in female ${ }^{11}$. Although this study focused on the migraine patients with comorbidities, it has been observed the similar female predominance. Reflection of this was also observed in distribution of occupation as most common profession of our participants was homemaking. A high frequency of severe migraine was observed in our participants. The frequency and duration of headache was comparable in participants with different comorbidities.

The association between anxiety and migraine was noted in both clinic and community-based studies ${ }^{12}$. The study conducted by McWilliams ${ }^{13}$ and his colleagues also showed the association between migraine and anxiety: $9.1 \%$ of subjects with migraine have GAD, compared to $2.5 \%$ of those without migraine. In the epidemiology cohort study ${ }^{14}$ in Zurich, the prevalence of GAD was high. The authors suggested that migraine with anxiety and depression may constitute a distinct syndrome comprising anxiety, often manifested in early childhood, followed by the occurrence of migraine headaches, and then by discrete episodes of depressive disorders in adulthood.

A high frequency of panic disorders was noted in patients with migraine and vise versa ${ }^{12}$. A population-based study evaluated the temporal relation of migraine and panic disorder ${ }^{15}$. In the study, the life-time prevalence of panic disorder was not only higher in subjects with migraine, but also in those with other severe headaches. In addition, the onset of panic disorder was significantly higher in subjects with migraine or severe headache ${ }^{12}$. The onset of migraine or severe headache was also more frequent in subjects with panic disorder. The study indicates a higher frequency of panic disorder was not specifically in migraine patients but also in other severe headache patients. A bidirectional temporal relationship was found between headache and panic disorder with a stronger direction of headache to panic disorder ${ }^{15}$.

\section{Conclusion}

Patients of migraine have comorbidities like GAD, MDD, obesity, hypertension and DM which need to be addressed appropriately for proper migraine headache management.

\section{References}

1. Ropper AH, Samuels MA. Headache and other craniofacial pains. In: Adams and Victors Principles of Neurology, 9th ed. McGraw Hill, New York, 2009;pp.162-188

2. Buse DC, Manack A, Serrano D, Turkel C, Lipton RB. Sociodemographic and comorbidity profiles of chronic migraine and episodic migraine sufferers. J neurol Neurosurg Psychiatr 2009;81:428-432

3. Breslau N, Davis GC, Schultz LR, Paterson EL. Migraine and major depression: A longitudinal study. Headache 1994;34:387-393

4. Scher AI, Bigal ME, Lipton RB. Comorbidity of migraine. Current Opinion in Neurology 2005;18:305-310

5. Headache Classification Subcommittee of the International Headache Society. The International Classification Of Headache disorders 2nd ed. Cephalalgia 2004; 24:1-160

6. Livibond PF, Lovibond SH. The structure of negative emotional states: comparison of the Depression Anxiety Stress Scales (DASS) with the beck depression and anxiety inventories 1995;33(3):335-43

7. Diagnostic and statistical manual of mental disorders. 4th ed. Washington DC, American Psychiatric Association. 2000;161-212 8. Chobanian AV, Bakris GL, Black HR, Cushman WC, Green LA, Izzo JL, et al. The Seventh Report of the Joint National Committee on Prevention, Detection, Evaluation and Treatment of High Blood Pressure. JAMA 2003;289:2560-72

9. Hanlon P, Byers M, Walker BR, Macdonald HM. Environmental and nutritional factors in disease. In: Colledge NR, Walker BR, Ralston SH, editors. Davidson's Principles \& Practice of 
Medicine. 21st ed. Churchill Livingstone 2010;116-25

10. Habib M, Alam B, Hoque A, Hoque B, Mohammad QD. Headache study of 3350 cases, Bangladesh Journal of Neuroscience 2001;17(1):1-5

11. Boes CJ, Capobianco DJ, Cutrer FM, Dodick DW, Garja I, Swanson JW. Headache and other craniofacial pain. In: Bradley WG, Daroff RB, Fenichel GM, Jankovic J, editors. Neurology in Clinical Practice.Vol II, 5th ed. Philadelphia: Butter worth-Heinemann; 2008;PP.2011-2062

12. Wang SJ, Fuh JL, Chen PK. Comorbidities of migraine.
Frontiers in Neurology 2010;1:16

13. McWilliams LA, Goodwin RD, Cox BJ. Depression and anxiety associated with three pain conditions: results from a nationally representative sample. Pain. 2004;111(1-2):77-83

14. Merikangas KR, Angst J, Isler H. Migraine and psychopathology: results of the Zurich cohort study of young adults. Archives of General Psychiatry. 1990;47(9):849-53

15. Breslau N, Schultz LR, Stewart WF, Lipton R, Welch KM. Headache types and panic disorder Directionality and specificity. Neurology. 2001;56(3):350-4 
